\title{
Balloon Observations of Auroral X-Rays in Canada I. Determination of Auroral X-Ray Illuminating Regions
}

\author{
Takamasa Yamagami, ${ }^{*}$ Masami Fujir, ${ }^{*}$ Jun Nishimura, ${ }^{*}$ \\ Hiroyuki Murakami, ${ }^{* *}$ Yo Hirasima, ${ }^{* *}$ Masao Kajiwara, ${ }^{* *}$ \\ Kiyoaki OKudaira, ${ }^{* *}$ and Masahiro Kodama*** \\ *Institute of Space and Aeronautical Science, \\ University of Tokyo, Tokyo, Japan \\ **Department of Physics, Rikkyo University \\ (St. Paul's University), Tokyo, Japan \\ ***The Institute of Physical and Chemical Research, \\ Tokyo, Japan
}

(Received October 5, 1977; Revised May 6, 1978)

Two balloons each carrying a payload consisting of three sets of $\mathrm{NaI}(\mathrm{Tl})$ scintillation counters have been launched from Thompson, Manitoba, Canada, in April 1975, to search precipitating mechanisms of auroral particles through observations of the bremsstrahlung X-rays in conjugation with the geo-stationary statellite ATS-6. Two omnidirectional counters, one of which had the modulation collimator, were used together with the directional counter inclined from the zenith. On the basis of the spin-modulated data obtained during the appearance of two distinct auroral X-ray bursts, two-dimensional source models, arc- and disc-shaped, are proposed for the auroral X-ray illuminating region. Also shown is an example of the eastward drift of another auroral X-ray source. Some of problems related to the analyses of data, i.e., background fluxes due to cosmic rays, and the response functions of the detectors are discussed.

\section{Introduction}

Morphological and dynamical studies of auroral X-rays have been developed step by step from a one-dimensional viewpoint to a three-dimensional one. In the earlier stage of studies, they were mainly based on balloon experiments which were carried out at a specified auroral zone site (e.g., Brown, 1966). In such a case, it is natural that most of studies were pointed to auroral phenomena which appeared within an extremely narrow limited region. In the next stage, a scheme of simultaneous multiple balloon flights from more than two stations were performed. This led us to the exploration of latitudinal and 
longitudinal distributions of the precipitating electrons from which auroral Xrays radiated, including the geomagnetic conjugacy between different hemispheres (Trefall and Brown, 1972; Treilhou, 1974; Barcus et al., 1973). Recently, this two-dimensional work is extended further toward the three-dimensional work directly related to the acceleration and propagation of auroral electrons, through joint work of balloon or rocket experiments with satellite observations (IMHOF, 1974).

As an approach to the three-dimensional studies, two balloons for auroral $\mathrm{X}$-ray measurements were launched from Thompson, Manitoba, Canada $\left(55.8^{\circ} \mathrm{N}\right.$, $97.9^{\circ} \mathrm{W}$ ) on April 8 and 9, 1975, when the geo-stationary satellite ATS-6 stayed on the magnetic line of force connected with the vicinity of Thompson. In order to trace clearly the precipitating region of electrons at balloon altitudes, three kinds of $\mathrm{NaI}$ scintillation counters were used in each flight.

So far, the spatial distribution of auroral X-rays was derived from two different experimental techniques. One is to use a set of two or more directional counter telescopes (GosLING, 1966; PARKS 1967) and another is an application of high spinning motion of a rocket to sky survey (VENKATESAN and Anger, 1975; Kodama and Oguti, 1976). In the present experiment, besides one omnidirectional counter and one directional counter, a third counter with the modulation collimator (BRADT et al., 1968; ODA, 1968) was used to discriminate the possible fine structure of the radiation region of auroral X-rays. They all were self-rotating at a spin rate of $3 \mathrm{rpm}$ in order to obtain spin-modulation data of X-rays. During the entire flight from midnight to dawn, there occurred a number of X-ray bursts whose fluxes ranged from 10 to $10^{4}$ times the background X-rays. In this report, only two typical events, one showing the greatest intensity enhancement and the other showing a distinct non-uniform spatial distribution of fluxes, were selected, and were analyzed so that the X-ray illuminating region could be identified. Descriptions are given here of the results of auroral X-rays thus obtained at balloon altitudes. Comparative studies with the results from the ATS-6 satellite will be made in the next paper.

\section{Instrumentation}

The principal parts of the balloon-borne instruments used in this work are shown in Fig. 1 and their specifications are summarized in Table 1. Three kinds of $\mathrm{NaI}$ scintillation counters, vertical, inclined and with modulation, were used.

a) Vertical counter The NaI crystal is of $1^{\prime \prime}$ in diameter and $1 \mathrm{~mm}$ thick, and a cylindrical collimator whose full opening angle is $70^{\circ}$ is attached to it pointing to the zenith. The collimator consists of concentric brass and tin cylinders, i.e., $1 \mathrm{~mm}$ thick brass cylinder inside and $1 \mathrm{~mm}$ thick tin cylinder outside. 



Fig. 1. Arrangement of principal parts of the balloonborne instruments and view-fields of the vertical and inclined counters.

Table 1. Balloon flights and auroral X-ray detectors.

\begin{tabular}{|c|c|c|c|c|c|c|}
\hline \multicolumn{3}{|c|}{ Ballon flights } & \multicolumn{4}{|c|}{ NaI scintillation counters } \\
\hline No. & Launch time & $\begin{array}{l}\text { Ceiling } \\
\text { altitude }\end{array}$ & Crystal size & $\begin{array}{l}\text { Mounting } \\
\text { angle }\left(^{\circ}\right)\end{array}$ & $\begin{array}{l}\text { Aperture } \\
\text { angle }\left(^{\circ}\right)\end{array}$ & $\begin{array}{c}\text { Energy } \\
\text { range }(\mathrm{keV})\end{array}$ \\
\hline \multirow{3}{*}{1} & \multirow{3}{*}{$\begin{array}{l}0346 \text { UT } \\
\text { April 8, } 1975\end{array}$} & \multirow{3}{*}{$13 \mathrm{mb}$} & 1: $1^{\prime \prime} \phi \times 1 \mathrm{~mm}$ & 0 & 70 & $15-85$ \\
\hline & & & 2: $1^{\prime \prime} \phi \times 1 \mathrm{~mm}$ & 17.5 & 35 & $15-85$ \\
\hline & & & 3: $1^{\prime \prime} \phi \times 1 \mathrm{~mm}^{1 \prime}$ & 0 & 70 & $15-84$ \\
\hline \multirow{3}{*}{2} & \multirow{3}{*}{$\begin{array}{l}0354 \text { UT } \\
\text { April 9, } 1975\end{array}$} & \multirow{3}{*}{$6 \mathrm{mb}$} & 1: $1^{\prime \prime} \phi \times 1 \mathrm{~mm}$ & 0 & 70 & $15-85$ \\
\hline & & & 2: $1^{\prime \prime} \phi \times 1 \mathrm{~mm}$ & 17.5 & 35 & $15-85$ \\
\hline & & & 3: $3^{\prime \prime} \phi \times 3 \mathrm{~mm}^{2 \prime}$ & 0 & 90 & $10-84$ \\
\hline
\end{tabular}

1) With $10^{\circ}$ pitch, 2-staged modulation collimator.

2) With $10^{\circ}$ pitch, 3-staged modulation collimator.

b) Inclined counter The NaI crystal of the same size and with the same collimator as in the vertical counter is mounted making an angle of $17.5^{\circ}$ from the zenith. The full opening angle of the collimator is $35^{\circ}$.

c) Modulation counter This is an X-ray detector equipped with a modulation collimator, which consists of two or three stages of parallel multi-wire grids, made of brass wire $2 \mathrm{~mm}$ in diameter. In the flight of April 8, 1975, a bi-grid modulation collimator was attached to the NaI crystal $1^{\prime \prime} \phi \times 1 \mathrm{~mm}$. Each stage was constructed of 30 parallel brass wires placed at intervals of $2 \mathrm{~mm}$, and they were spaced $20 \mathrm{~mm}$. In the second flight of April 9, the collimator constructed of three stages of grids spaced $30 \mathrm{~mm}$ each was mounted on the $\mathrm{NaI}$ crystal $3^{\prime \prime} \phi \times 3 \mathrm{~mm}$. The modulation collimator has in principle multiple optical 


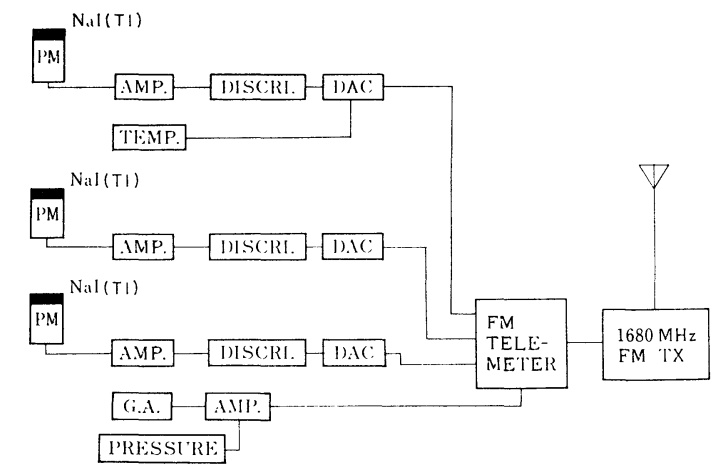

Fig. 2. A block diagram of the electronic circuits of the payload.

transmission bands of triangular shapes. The band spacing, or, the base length of the triangle was such that the fine structure of the auroral X-ray source can be discriminated with an angular resolution of $10^{\circ}$, though this resolution of angle is not necessarily sufficient. In the case of two-stage colimator, the transmission bands appear successively for an X-ray source, while alternate patterns of bands appears in the case of the three-stage collimator.

The above three kinds of counters were installed inside one gondola, which was rotated around its vertical axis at a rate of $3 \mathrm{rpm}$. The azimuthal direction changing with time was determined from records of a geomagnetic aspect-meter operated simultaneously.

The upper and lower discriminator levels of the associated electronic circuits were taken so as to give the energy ranges of X-rays as described in Table 1 . In order to count individual pulses from the counters digitally, the technique of the stepcount scaler developed by OGAWARA and HAYASHI (1971) was introduced. A block diagram of the electronic circuit is shown in Fig. 2. After the number of counter pulses was scaled down by $1 / 2^{3}$ for the modulation counter and by $1 / 2^{5}$ for the other two, it was converted to analogue signals and was input to a 1,680 $\mathrm{MHz}$ FM/FM transmitter.

\section{Balloon Flights and Auroral X-Ray Activities}

The ballons were launched from Thompson on 0346 UT (2116 LT) on April 8, 1975 and on 0354 UT (2124 LT) on April 9, respectively. The first one (named Flight No. 1) flew for about $10 \mathrm{hr}$ after reaching a ceiling altitude of $13 \mathrm{mb}$. The second (Flight No. 2) reached $6 \mathrm{mb}$ ceiling and succeeded in making an 11-hr flight, during which a number of interesting events of auroral X-ray bursts were detected.

Time profiles of 1-min counting rates obtained in Flights No. 1 and No. 2 


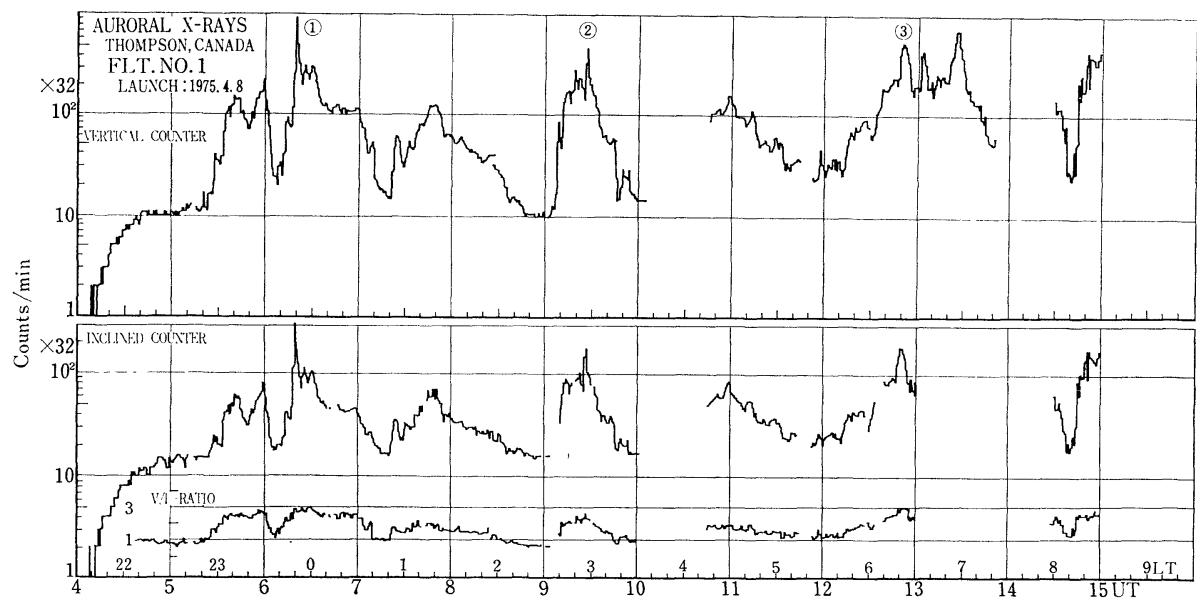

Fig. 3. Intensity-time profiles of auroral X-rays observed in Flight No. 1. 1-min counting rates from the vertical and the inclined counters are plotted, together with the counting rate ratio between the two counters. LT: at Thompson.

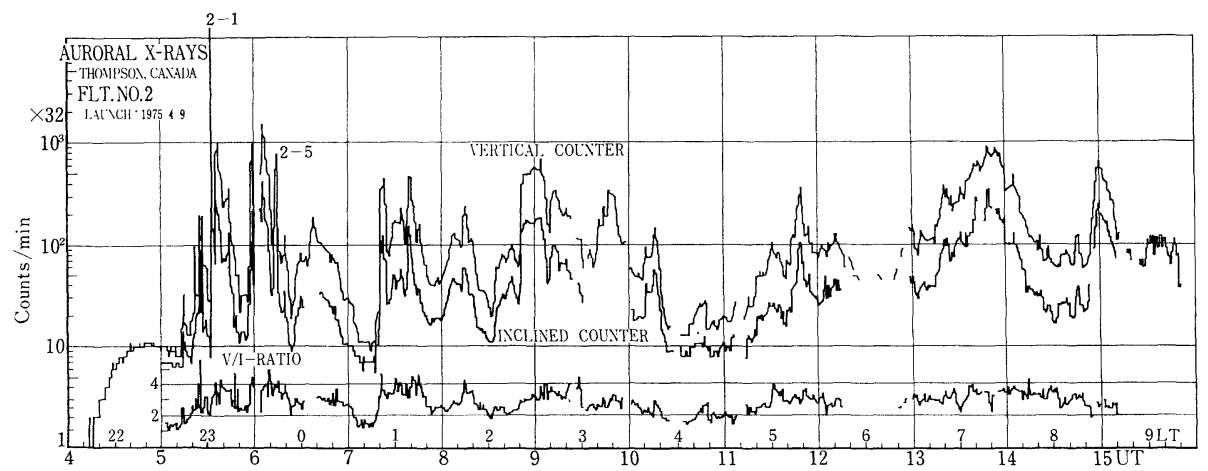

Fig. 4. Intensity-time profiles of auroral X-rays observed in Flight No. 2. 1-min counting rates from the vertical and the inclined counters are plotted, together with the counting rate ratio between the two counters.

are shown in Figs. 3 and 4, respectively. It is evident from these figures that overall activities of auroral X-rays were relatively high during both flights, particularly in Flight No. 2. Among a number of distinct X-ray bursts, an event showing the greatest enhancement and another one suggesting a typical nonuniform distribution of flux in the sky were selected, and were named Event 2-1 and Event 2-5 respectively. To see easily the non-uniformity of the flux, the ratio of the counting rate from the vertical counter to that from the inclined counter is shown in the lower portions of Figs. 3 and 4. This ratio is a measure of the activity of auroral X-rays coming from a specified direction against those 
coming uniformly from all sky. Therefore, this ratio, or its reciprocal, during Events 2-1 and 2-5 will be examined.

Although the auroral X-ray bursts appeared many times throughout the whole flight periods of April 8 and 9, the counting rates decreased occasionally to the normal background level due to cosmic rays. For example, the counting rates of both the vertical and inclined counters show the normal level at around 0505 UT and 0710 UT in Flight No. 2. At these quiet times, the counting rate of the vertical counter is 5.4 counts/sec, when the ratio of counts of the inclined counter to that of the vertical counter (hereafter denoted by $I / V$ ratio $r$ ) is 0.69 and the counting rate of the modulation counter is 12.0 counts/sec.

The reliability of these observed background intensities will now be examined. In the case of the vertical counter, the product of its effective area $S$ and full opening solid angle $\Omega$ is given by $S \Omega=2.2 \mathrm{~cm}^{2} \mathrm{str}$, so that the background flux in the energy range of $15-85 \mathrm{keV}$ is $2.5 \mathrm{~cm}^{-2} \mathrm{sec}^{-1} \mathrm{str}^{-1}$. On the other hand, the background X-ray flux measured by Peterson (1969) at $40^{\circ} \mathrm{N}$ geomagnetic latitude and at a depth of $3.5 \mathrm{~g} \mathrm{~cm}^{-2}$ is $1.6 \mathrm{~cm}^{-2} \mathrm{sec}^{-1} \mathrm{str}^{-1}$ in the same energy range. It is found that both background fluxes are consistent with each other, considering the latitude and altitude dependences of cosmic ray intensity.

Next, the contribution of the bremsstrahlung to the background was estimated. The bremsstrahlung X-rays arise in the collimator materials due to energetic electrons precipitating into the atmosphere. For the vertical counter, the counting rate due to this process was estimated to be $10^{-2}$ counts/sec (see Appendix I), being much smaller than the observed background counts of 5.4 counts/sec. Consequently, there is little contribution of the bremsstrahlung Xrays generated in the collimator.

Possible scattering of X-rays in the atmosphere will obscure the real image of an X-ray source, if the balloon altitude is not high enough. To estimate this effect quantitatively, one of the authors (OKUDAIRA, 1979) has analyzed its order of magnitude theoretically. According to his calculated results, this effect is not serious under the present experimental condition. For example, if the vertical incidence of photons is assumed at the top of the atmosphere, Thomson scattering enhances the count of the vertical counter by about $12 \%$ at a depth of $1.5 \lambda\left(5.6 \mathrm{~g} \mathrm{~cm}^{-2}\right)$, where $\lambda$ is the attenuation mean free path of photons, and also the $I / V$ ratio $r$ changes from zero to about 0.03 .

\section{Results of Observation}

\subsection{Event 2-1 on April 9, 1975}

During the period from 0532:17 UT to 0532:39.4 UT on April 9, 1975, 


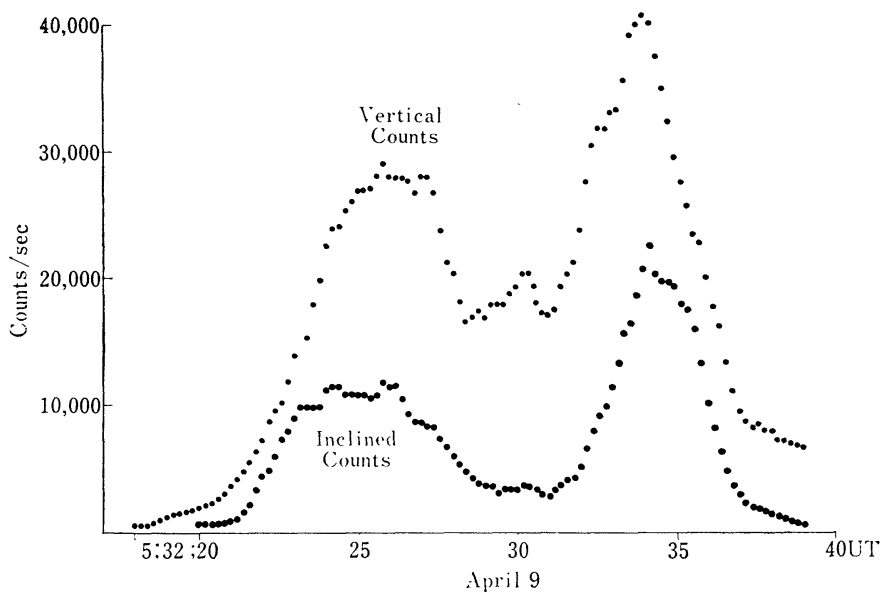

Fig. 5. Time variation of auroral X-ray intensities observed in Event 2-1. 0.2-sec counts from the vertical and the inclined counters are plotted.

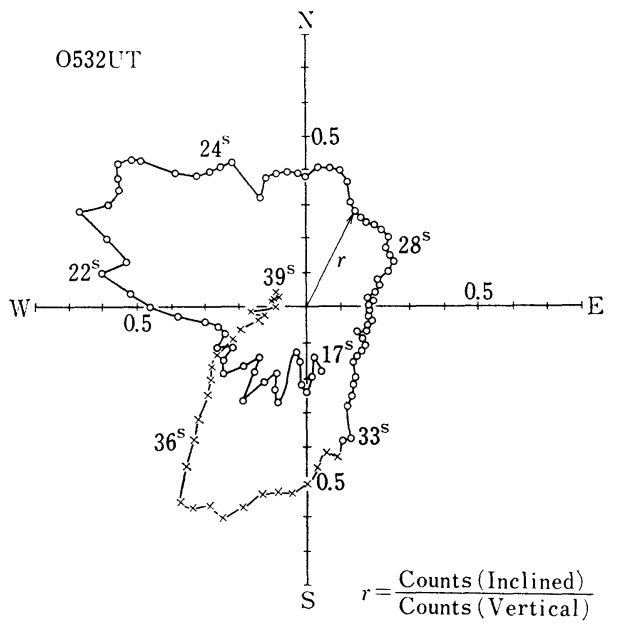

Fig. 6. Counting rate ratio $r$ against azimuthal angle of viewing direction of the inclined counter in the case of Event 2-1.

the greatest enhancement in the counting rate was recorded. To examine the details of its time variations, counts were read out every $0.2 \mathrm{sec}$ and are plotted in Fig. 5. At each of the double peaks, the fluxes were about $10^{4}$ times the background flux. For the modulation counter, however, no reliable record was obtained, because the actual counting rate was too high to be recorded on the magnetic tape data recorder available which had a limited frequency response.

In Fig. 6 are plotted the $I / V$ ratios $r$ for every $0.2 \mathrm{sec}$ on the geographic polar coordinate centered at the balloon location. It is evident that an enhancement of the $r$ values was seen predominantly in two azimuthal directions: a NW 
Table 2. Selected data of auroral X-rays observed in Event 2-1.

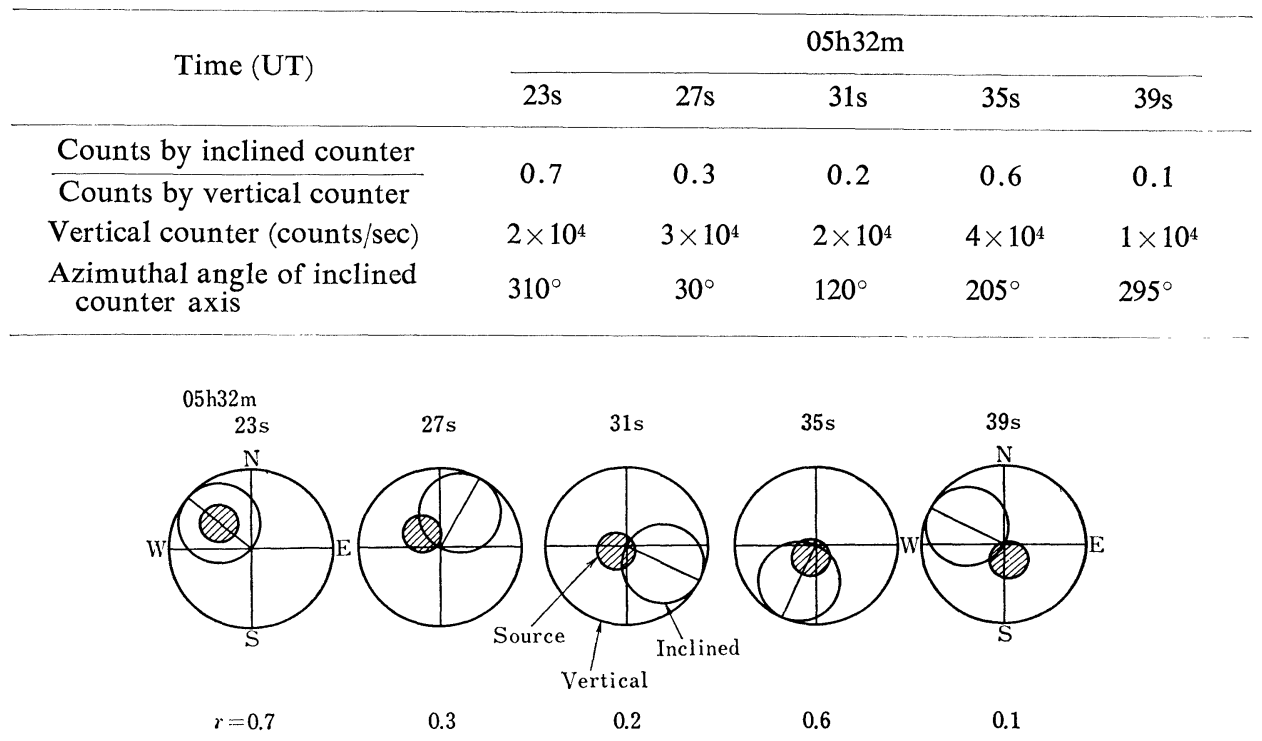

Fig. 7. Spatial drift of the auroral X-ray source as indicated by hatched circle during Event 2-1. Two circular areas, smaller and larger, are radial viewing fields of the inclined and vertical counters, respectively.

direction of about $310^{\circ}$ at $0532: 23 \mathrm{UT}$ and a SSW direction of about $210^{\circ}$ at $0532: 35.5 \mathrm{UT}$. It is possible to give two different interpretations for this fact. One is that two different auroral X-ray sources appeared independently in different directions. Another is that one X-ray source drifted from the NW direction to the SSW direction. Numerical values of observation parameters obtained at every $4 \mathrm{sec}$ during the time $5^{\mathrm{h}} 32^{\mathrm{m}} 23^{\mathrm{s}}-39^{\mathrm{s}}$ are listed in Table 2 . It is clearly seen from Fig. 6 and Table 2 that the counting rate of the vertical counter changed by a factor of 4 , from $1 \times 10^{4}$ to $4 \times 10^{4}$, but the $r$ value changed from 0.7 at the maximum to 0.1 at the minimum, or, by a factor of 7 . Such different amplitudes of time variations are more favorable for the drift model of the X-ray source, as schematically illustrated in Fig. 7.

\subsection{Event 2-5 on April 9, 1975}

Time variations of $1-\mathrm{sec}$ counts of the vertical and inclined counters during the period from 0613:12 UT to 0615:12 UT are shown in Fig. 8, and also the $r$ values in the same time interval are plotted in Fig. 9. It is of interest that the $r$ values were remarkably enhanced whenever the viewing direction of the inclined counter pointed to the south.

Figure 10 shows the ratio of the counting rates of the modulation counter 


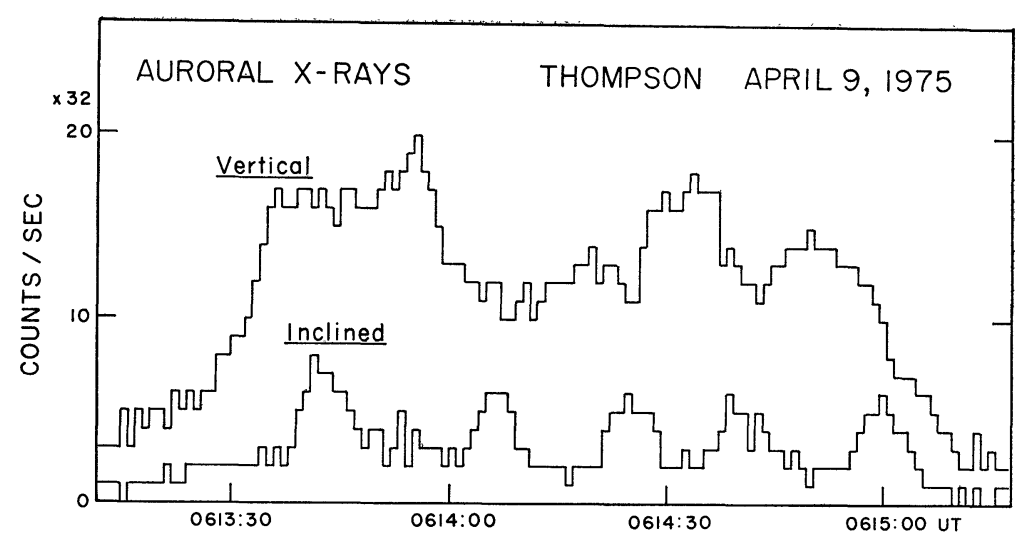

Fig. 8. Time variation of auroral X-ray intensities measured by the vertical and inclined counters in Event 2-5.

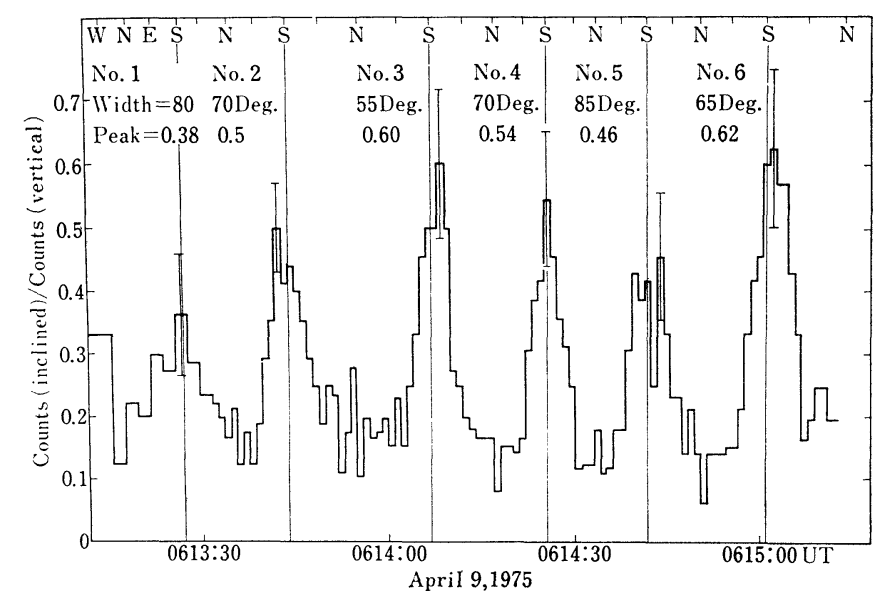

Fig. 9. Time variation of the counting rate ratio $r$ in Event 2-5.

to those of the vertical counter (denoted by $r_{\mathrm{m}}$ ). Similar patterns of variations as in Fig. 9, that is, the periodical enhancement in the south can be again recognized. In principle, however, a pattern of enhancement also should appear in the opposite direction, north, if there is a specified X-ray source in the southern direction. It is because the modulation collimator used here consists of parallel multi-wires and is plane-symmetrical. One of the reasons for the disappearance of such enhancement in the north might be attributed to a possible change of the counter sensitivity due to the change of the optical contact of the NaI crystal to the surface of the photomultiplier tube during the flight. 


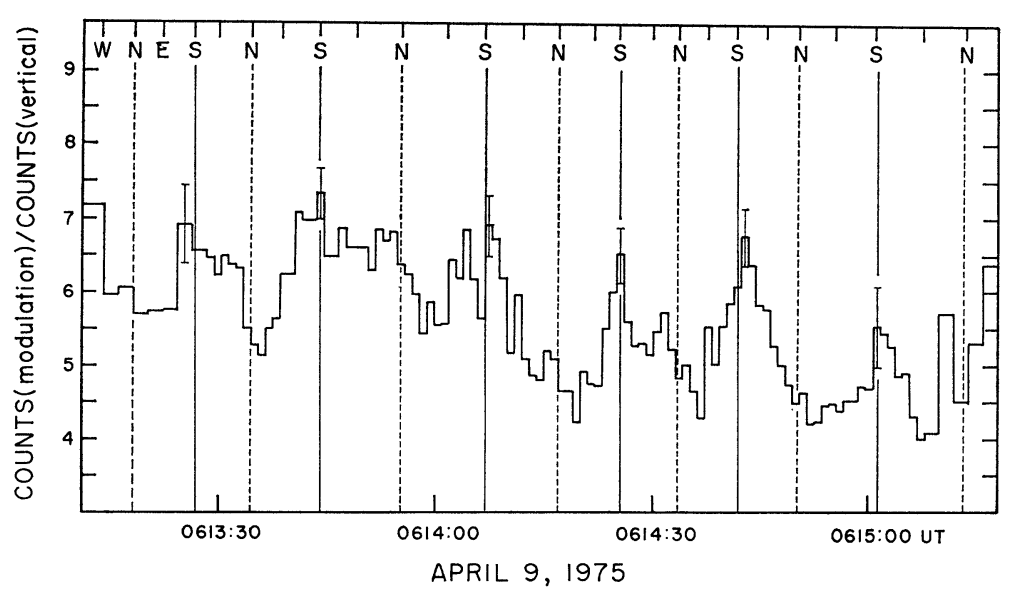

Fig. 10. Time variation of the counting rate ratio $r_{\mathrm{m}}$ of the modulation counter to the vertical counter in Event 2-5.

\section{Determination of X-Ray Source Region}

To interpret the periodical fluctuations of the $r$ and $r_{\mathrm{m}}$ values shown in Figs. 9 and 10 of Event 2-5 in terms of the source function such as the shape, orientation, and intensity, two tentative source models are introduced and compared with the observed results.

\subsection{Discoid model with the uniform source intensity-Model 1}

First, it is assumed that the source is discoid in shape and the source intensity is uniform within the disc. The direction of the source is expressed by the zenith angle $\theta$ and the azimuthal angle $\phi$. The source size is given by the radial angle $\Phi_{0}$ viewed from the observation point. Simulation calculations were performed on this idealized model by assuming various sets of values for the parameters $\theta, \phi$ and $\Phi_{0}$. The best fitting values were then determined by comparing the calculated results with the observed ones. In carrying out the calculations, the response function of a counter with a cylindrical collimator to a point source was first calculated and then the computation was extended to the discoid source. Descriptions of these calculations will be given in Appendix II.

The response function of the vertical counter is independent of the azimuthal angle $\phi$, while that of the inclined counter is not. Accordingly, the $r$ values are expressed as a function of $\phi$. Figure 11 shows results of the calculation of $r$ as functions of $\theta$ and $\Phi_{0}$, where the half width of the azimuthal angle at half maximum of $r$ (HWHM) was taken as the ordinate. On the other hand, when the observed HWHMs of the $r$ values for each of six peaks appearing in Fig. 9 


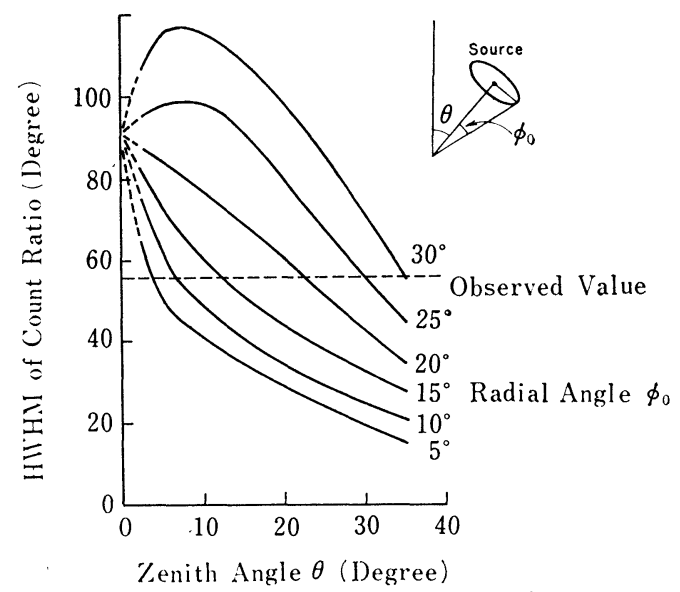

Fig. 11. Response functions of the inclined counter as a function of zenith angle in the case of discoid source Model 1. The ordinate gives the HWHM, in unit of degree, of the azimuthal angle corresponding to a half of the counting rate ratio $r$.

are averaged, the value of $55^{\circ}$ is obtained. This also is indicated by the dashed line in Fig. 11. Thus, intersections of the individual solid lines with the dashed one in Fig. 11 should give some possible solutions for the unknown source function. This consideration is, however, limited to discussions about the source size alone.

Now we could estimate the source function concerning the intensity itself. Another parameter defining the periodical variation in Fig. 9 is the peak-tovalley ratio of the $r$ vaiue. It was derived in a similar way as functions of $\theta$ and $\Phi_{0}$. From the results thereby obtained and the above-mentioned consideration of Fig. 11, it is concluded that the following two pairs of $\theta$ and $\Phi_{0}$ are favorably accepted for the source function:

$$
\begin{array}{lll}
\theta=35^{\circ} \pm 5^{\circ} & \text { and } & \Phi_{0}=30^{\circ} \pm 5^{\circ}, \\
\theta=10^{\circ} \pm 5^{\circ} & \text { and } & \Phi_{0}=10^{\circ} \pm 5^{\circ},
\end{array}
$$

where the errors are those due to the limited number of calculations which were done in $5^{\circ}$ step in the angle.

From Fig. 9, we obtain as the average values

$$
\frac{N_{\mathrm{I}}}{N_{\mathrm{V}}+S_{\mathrm{V}}}=0.15,
$$

and 


$$
\frac{S_{\mathrm{I}}}{N_{\mathrm{V}}+S_{\mathrm{V}}}=0.10,
$$

where $N_{\mathrm{I}}$ is the background counts measured by the inclined counter, $N_{\mathrm{V}}$ the background counts measured by the vertical counter, $S_{\mathrm{I}}$ the auroral X-ray counts measured by the inclined counter, and $S_{\mathrm{V}}$ the auroral X-ray counts measured by the vertical counter. In general, the relationship between the vertical and inclined counters is expressed briefly by

and

$$
N_{\mathrm{I}}=\alpha N_{\mathrm{v}},
$$

$$
S_{\mathrm{I}}=\beta S_{\mathrm{V}} \text {. }
$$

When the parameters $\alpha$ and $\beta$ are calculated for a point source, their upper and lower limits are found to be 0.33 and zero, respectively, taking into account the response functions (see Appendix III). On the other hand, the simulation calculations of $\alpha$ for the source models i) and ii) give 0.25 and 0.4 respectively. Since the latter value of 0.4 is out of the above limitation, it is to be said that the source model i) is more preferable.

\subsection{Arc-shaped model with the Gaussian intensity distribution-Model 2}

Another source model is examined in which the auroral X-ray source is arc-like in shape with the Gaussian distribution of the intensity. The function denoting this source is given by

$$
f(\theta, \phi)=f_{0}(\theta) \exp \left\{-\frac{\phi^{2}}{b(\theta)^{2}}\right\} .
$$

If the response function of the inclined counter as described in Appendix II is approximated by

$$
\alpha(\theta, \phi)=\alpha_{0}(\theta) \exp \left\{-\frac{\phi^{2}}{a(\theta)^{2}}\right\},
$$

then the ratio $r$ is approximated again by the convolution of the Gaussian distributions, that is,

$$
r(\theta, \phi)=r_{0}(\theta) \exp \left\{-\frac{\phi^{2}}{c(\theta)^{2}}\right\},
$$

so that we can determine the width of the source function from the equation,

$$
b(\theta)=\left\{c(\theta)^{2}-a(\theta)^{2}\right\}^{1 / 2} .
$$

Now, the ratio $r$ can be defined by the expression,

$$
r=\frac{\int \alpha\left(\theta, \phi^{\prime}-\phi\right) f(\theta, \phi) \mathrm{d} \phi}{\int f(\theta, \phi) \mathrm{d} \phi},
$$


Table 3. Various quantities of the arc-shaped source Model 2.

\begin{tabular}{lcccccc}
\hline \multicolumn{1}{c}{ No. } & 1 & 2 & 3 & 4 & 5 & 6 \\
\hline \begin{tabular}{l}
$0.25 N$ \\
\hline$S+N$
\end{tabular} & 0.2 & 0.15 & 0.15 & 0.15 & 0.15 & 0.15 \\
$\frac{N+S}{S}$ & 5 & 2.5 & 2.5 & 2.5 & 2.5 & 2.5 \\
$\int r \mathrm{~d} \phi$ & 20 & 44.1 & 46.8 & 45 & 46.8 & 45 \\
$\int \alpha \mathrm{d} \phi$ & 100 & 110 & 117 & 112 & 117 & 112 \\
Peak value $P$ & 0.21 & 0.35 & 0.45 & 0.40 & 0.30 & 0.45 \\
Observed width $c$ & 80 & 70 & 55 & 70 & 80 & 65 \\
Response width $a$ for $\bar{\theta}=22^{\circ}$ & 32 & 32 & 32 & 32 & 32 & 32 \\
$P c$ & 16.8 & 24.5 & 24.8 & 28 & 24 & 29 \\
$\frac{N+S}{S}$ Pc & 84 & 61.3 & 62 & 70 & 60 & 72.5 \\
Source width $b$ & 73 & 62 & 45 & 62 & 73 & 57 \\
\hline
\end{tabular}

$N$ and $S$ are equivalent to $N_{V} S_{\mathrm{V}}$ used in Eq. (1), respectively.

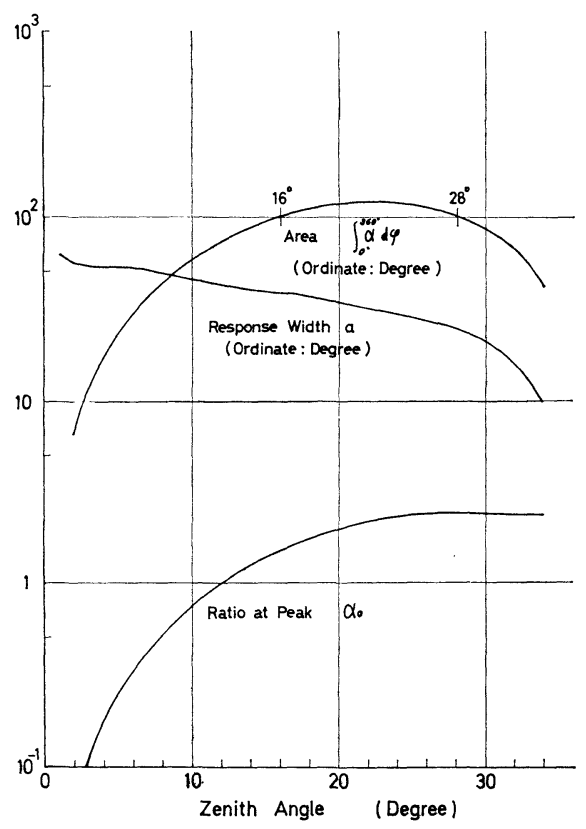

Fig. 12. Response functions of the inclined counter as function of zenith angle in the case of arc-shaped source Model 2. 
when a point source appears in an arbitrary azimuthal direction $\phi^{\prime}$. Therefore, we have

$$
\int_{0^{\circ}}^{360^{\circ}} r d \phi^{\prime}=\int_{0^{\circ}}^{360^{\circ}} \alpha\left(\theta, \phi^{\prime}\right) \mathrm{d} \phi^{\prime} \equiv \bar{\alpha}(\theta) .
$$

The values of $\bar{\alpha}(\theta)$ are shown in Fig. 12, together with values of $a$ and $\alpha_{0}$. Since the experimental values corresponding to $\bar{\alpha}$ are obtained from Fig. 9, we can determine the effective zenith angle of the arc-like X-ray source. Values of various quantities obtained from the observed results given in Fig. 9 are summarized for the six peaks in Table 3, in which P gives the peak value of $r$. From $\bar{\alpha}(\theta)$ in Fig. 12, it is seen that the source appeared in the zenith angle lying in the range from $16^{\circ}$ to $28^{\circ}$. Corresponding to this median value of $22^{\circ}$, we obtain $a\left(22^{\circ}\right)=$ $32^{\circ}$. The $1 / \mathrm{e}$ width $b(\theta)$ of the source taken along the azimuthal direction is given by Eq. (6), as both the response $1 / \mathrm{e}$ width $a(\theta)$ and the observed $1 / \mathrm{e}$ width $c(\theta)$ are known. The results are shown in Table 3 ; the averaged value of $b$ is derived from the table and is found to be $62^{\circ}$. The uncertainties included in the zenith and azimuthal angles are found to be $6^{\circ}$ and $5^{\circ}$, respectively. The latter came from the fluctuation of observed $r$, which is roughly 0.1 . Consequently, the source parameters for Model 2 are given as follows:

and

$$
\begin{aligned}
& \phi=180^{\circ} \pm 5^{\circ} \text { (southward), } \\
& \bar{\theta}=22^{\circ} \pm 6^{\circ},
\end{aligned}
$$

$$
\bar{b}=62^{\circ} \pm 5^{\circ}
$$

\subsection{Analysis of the data from the modulation counter}

In the present experiment, the modulation counter as well as the vertical and inclined counter was rotated at a spin rate of $3 \mathrm{rpm}$. Although the onedimensional modulation collimator was actually used, the two-dimensional scanning became possible by the operation of the self-rotation of the collimator. The response function of the modulation counter to parallel X-ray beam was calculated as a function of azimuthal and zenith angles. Some examples of transparencies of X-rays thus obtained are presented in Fig. 13. The value of $r_{\mathrm{m}}$ was then calculated for Model 1. Two examples of calculations are shown in Fig. 14, for two cases of possible source functions which were inferred from the analyses of $r$. Comparison of the calculated result (Fig. 14) with the observed one (Fig. 10) suggests that the source function represented by $\theta=35^{\circ}$ and $\phi_{0}=$ $30^{\circ}$ is in better fitting. This is also consistent with the results of $\alpha$ and $\beta$ deduced from the observation by the inclined counter. In the case of another solution, in which $\theta=10^{\circ}$ and $\phi_{0}=10^{\circ}$, the calculated $r_{\mathrm{m}}$ values are somewhat larger than the observed ones and give smaller amplitude of variation. 


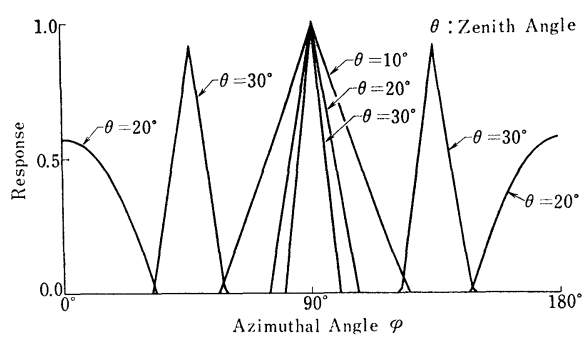

Fig. 13. Response functions of the modulation counter as a function of azimuthal angle for a point source.

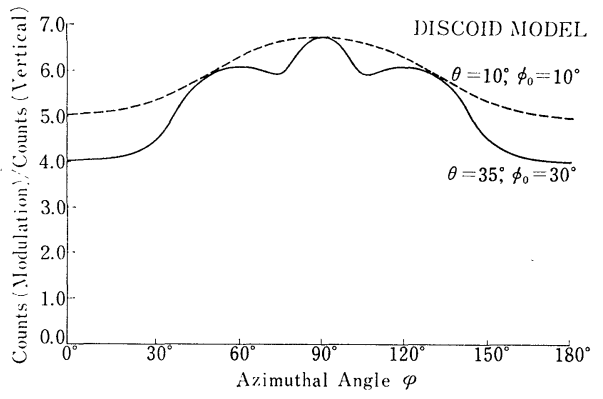

Fig. 14. Response functions of the modulation counter as a function of azimuthal angle in the case of discoid source Model 1. Calculated results for different two source functions are shown.

\section{Discussion and Summary}

Two distinct events of auroral X-ray bursts showing different characters, named Event 2-1 and Event 2-5, were observed at a level of $6 \mathrm{mb}$ over Thompson on 0532: 17 UT-0532: 39.4 UT and 0613:12 UT-0615: 12 UT of April 9, 1975, respectively. The former is specified by a drift of the X-ray source with constant velocity, whereas the latter by a stationary pattern of X-ray source.

Event 2-1 The time variation of the $r$ values given in Fig. 6 could be well understood by assuming an approximately eastward movement of a definite area of the $\mathrm{X}$-ray source, which is shown schematically by the sequence of hatched circles in Fig. 7 . If $100 \mathrm{~km}$ is taken to be the average altitude of the production layer of auroral X-rays, we obtain the source diameter of about 20 $\mathrm{km}$. The result of observation shows that such an X-ray source moved from NW to SE with a constant velocity of $2 \mathrm{~km} / \mathrm{sec}$ or so.

Event 2-5 To interpret the periodic variation of the auroral X-ray intensity ratio $r$ shown in Fig. 9, two tentative models of the X-ray source, the disctype and arc-type, have been examined, in connection with its size and direction 


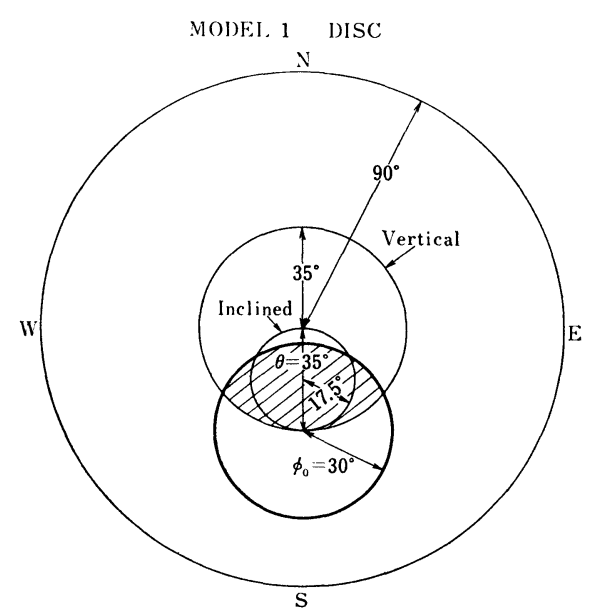

(a)

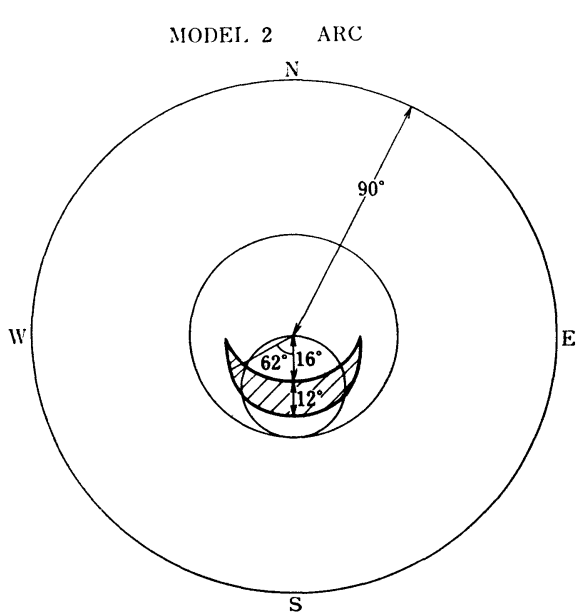

(b)

Fig. 15. Direction and size of the auroral X-ray source inferred from X-ray observations under an assumption of Model 1 or 2 .

of appearance. Regardless of the difference in the source shape between the two models, the center of the source is found in the same azimuthal direction $180^{\circ} \pm$ $5^{\circ}$, as seen in Fig. 15. Auroral X-rays from the hatched area can contribute to the counting rates of the vertical and inclined counters, since part of the sky whose zenith angle is geater than $35^{\circ}$ is out of the viewfield of both counters. The azimuthal direction and dimension of the source within the viewfield are not so different between the two models. The zenith angles are $35^{\circ} \pm 5^{\circ}$ and $16^{\circ}-28^{\circ}$ for the discoid and arc models, respectively. Assuming $100 \mathrm{~km}$ again to be the auroral altitude, the radius of the discoid source estimated from the viewing half angle of $30^{\circ} \pm 5^{\circ}$ turns out to be $40 \pm 6 \mathrm{~km}$. If the arc source is assumed, its width is given by $124^{\circ} \pm 10^{\circ}$, that is, $57 \pm 5 \mathrm{~km}$, when the $X$-ray intensity is assumed to obey the $1 / \mathrm{e}$ law toward the azimuthal direction.

Generally speaking, like the optical auroral pattern the auroral X-ray source is not stable in time nor spatially uniform but occasionally could be deformed with some fine structures. Hence, the above results may give only the overall character of the source. In the case of Event 2-5 is found a particular interest of the stable entry of high energy electrons, by which a constant inflow of auroral X-rays from a specified direction was caused during the 2-min interval. Though such a stable electron precipitation seems to be from a certain equatorial region geomagnetically connected with the location of the Thompson district, it is unreasonable to consider that an auroral electron source continued to stay in the equatorial region during that time. Transient supply of electrons by the convection mode may be a more preferable interpretation. In association 
with the other X-ray event occurred during the present flight, there is an experimental evidence given by ATS-6 satellite to support transient supplies of several ten $\mathrm{keV}$ electrons to the conjugate region assumed. Details of this field of study will be reported in the next paper Part II.

The authors would like to express their profound appreciation to Professor K.C. Clark, University of Washington, for his arrangement of the Japan-U.S. cooperation project, and to Professor G.K. Parks, University of Washington, for conducting the experiment and supplying the balloon flight equipment. Thanks are also due to the auroral X-ray study group of University of Washington. The authors are also grateful to the staff of the Computer Center, University of Tokyo, and the staff of the Computer Center, Rikkyo University, for the use of the computer facilities.

\section{Appendix I. Background X-Rays}

Background X-rays mean X-rays entering directly from the outside of the gondola to the counter and also the bremsstrahlung $\mathrm{X}$-rays emitted by the interaction of cosmic ray electrons with the gondola material, counter collimator and so on, when no auroral phenomena appear. The former includes the atmospheric and the diffuse cosmic X-rays.

As for the diffuse cosmic X-rays, many investigations have been performed in the field of X-ray astronomy (e.g., Horstman et al., 1975). Using their energy spectra reported so far, the contribution to the counting rate of the present vertical counter is estimated to be 0.5 counts $/ \mathrm{sec}$ at the depth of $6 \mathrm{~g} \mathrm{~cm}^{-2}$ in our corresponding energy range of $15-85 \mathrm{keV}$. The contribution of the diffuse cosmic $\mathrm{X}$-rays is, therefore, only $9 \%$ of the total background fluxes observed.

Let us next estimate the contribution of the bremsstrahlung X-rays generated in the collimator. The parent electron fluxes were extrapolated from their energy spectrum in the energy range over $4 \mathrm{MeV}$. This spectrum was that calculated theoretically using the energy spectra of primary cosmic rays and also was the spectrum confirmed by electron observations at the quiet time in Fort Churchill (Beuerman, 1971). The counting rate of the bremsstrahlung X-rays in the collimator consisting of brass and tin was then calculated using the probability function of Bethe and Heitler (Rossi, 1952). The calculated results gave the bremsstrahlung X-ray fluxes of about $10^{-2}$ counts/sec, being negligibly small in comparison with the total background of 5.4 counts $/ \mathrm{sec}$. The main part of X-rays, that is, background X-rays are explained only by the atmospheric component.

If the intensity enhancement of energetic electrons occurs with auroral activity, it is natural that a greater contribution by this process comes in. Also, if there exists much more material in the gondola, the background X-rays due to 
this process will increase considerably even by an order of magnitude. For example, if there exists an aluminum block of $100 \mathrm{~cm}^{2} \times 3 \mathrm{~cm}$ within a solid angle of $\sim 1$ steradian from the counter, the bremsstrahlung X-ray flux generated in this material amounts to about 0.2 counts/sec. It still is much smaller than the observed total background fluxes. Such an effect, however, would not be negligible if much more material is present in the vicinity of the counter.

Appendix II. Response Functions of a Directional Counter with a Cylindrical Collimator

1) Discoid source model

We denote the effective radius of the NaI scintillation counter by $r_{1}$, and the inner radius and the length of the attached cylindrical collimator, which is inclined at an angle $\eta$ from the zenith, by $r_{2}$ and $h$, respectively. Then, the response function of this counter can be deduced by the method of spherical trigonometry for the discoid X-ray source having a uniform intensity distribution and radial angle $\Phi_{0}$ in a direction $(\theta, \phi)$. That is,

$$
R\left(\theta, \phi, \Phi_{0}\right)=\frac{2}{\pi r_{1}^{2}} \int_{\delta_{1}}^{\delta_{2}} s(\delta) \cdot \cos \delta \cdot a(\delta) \cdot \sin \delta \cdot \mathrm{d} \delta
$$

Here, $\delta$ is the deflection angle measured from the collimator axis, and $s(\delta)$ and $a(\delta)$ are given by

$$
\begin{gathered}
s(\delta)=\frac{\pi}{2}\left(r_{1}^{2}+r_{2}^{2}\right)-x_{1} \sqrt{\left(r_{1}^{2}-x_{1}^{2}\right)}+\left(x_{1}-x_{0}\right) \sqrt{\left\{r_{2}^{2}-\left(x_{1}-x_{0}\right)^{2}\right\}} \\
-r_{1}^{2} \sin ^{-1} \frac{x_{1}}{r_{1}}+r_{2}^{2} \sin ^{-1} \frac{x_{1}-x_{0}}{r^{2}}, \\
\left(x_{0}=h \tan \delta, \quad x_{1}=\frac{r_{1}^{2}-r_{2}^{2}+x_{0}^{2}}{2 x_{0}}\right), \\
a(\delta)=\cos ^{-1} \frac{1}{\sin \delta \tan \chi}\left(\frac{\cos \Phi_{0}}{\cos \chi}-\cos \delta\right), \\
\left(\chi=\cos ^{-1}(\cos \theta \cos \eta+\sin \theta \sin \eta \cos \phi)\right) .
\end{gathered}
$$

The lower and upper limits of integration are

$$
\begin{aligned}
& \delta_{1}=\cos ^{-1}(\cos \theta \cos \eta+\sin \theta \sin \eta \cos \phi)-\Phi_{0}, \\
& \delta_{2}=\cos ^{-1}(\cos \theta \cos \eta+\sin \theta \sin \eta \cos \phi)+\Phi_{0} .
\end{aligned}
$$

2) Arc-shaped source model

As already described, the ratio $r$ is expressed by the Gaussian type function as follows: 


$$
\begin{aligned}
r & =r_{0}(\theta) \exp \left\{-\frac{\phi^{2}}{c(\theta)^{2}}\right\} \\
& =\frac{S}{N+S} \alpha_{0} \frac{a}{c} \exp \left\{-\frac{\phi^{2}}{c(\theta)^{2}}\right\},
\end{aligned}
$$

where $c^{2}=a^{2}+b^{2}$ and $b$ is the source width. Denoting the peak value of the observed $r$ by $P\left(P \equiv r_{0}\right)$, we obtain by integration of Eq. (A2)

$$
\int_{0^{\circ}}^{360^{\circ}} P \exp \left(-\frac{\phi^{2}}{c^{2}}\right) \mathrm{d} \phi=\frac{S}{N+S} \int_{0^{\circ}}^{360^{\circ}} \alpha_{0} \frac{a}{c} \exp \left(-\frac{\phi^{2}}{c^{2}}\right) \mathrm{d} \phi
$$

and then,

$$
P=\frac{S}{N+S} \alpha_{0} \frac{a}{c} .
$$

$((N+S) / S) P c$ given in Table 3 should, therefore, be identical to the product of the response width $a(\theta)$ and its peak value $\alpha_{0}(\theta)$, if an appropriate value is assigned to the zenith angle $\theta$. Both quantities actually coincide with each other in the range of $\theta$ from $16^{\circ}$ to $28^{\circ}$.

Appendix III. Allowed Regions of $\alpha$ and $\beta$

There are obvious limitations for the values taken by parameters $\alpha$ and $\beta$.

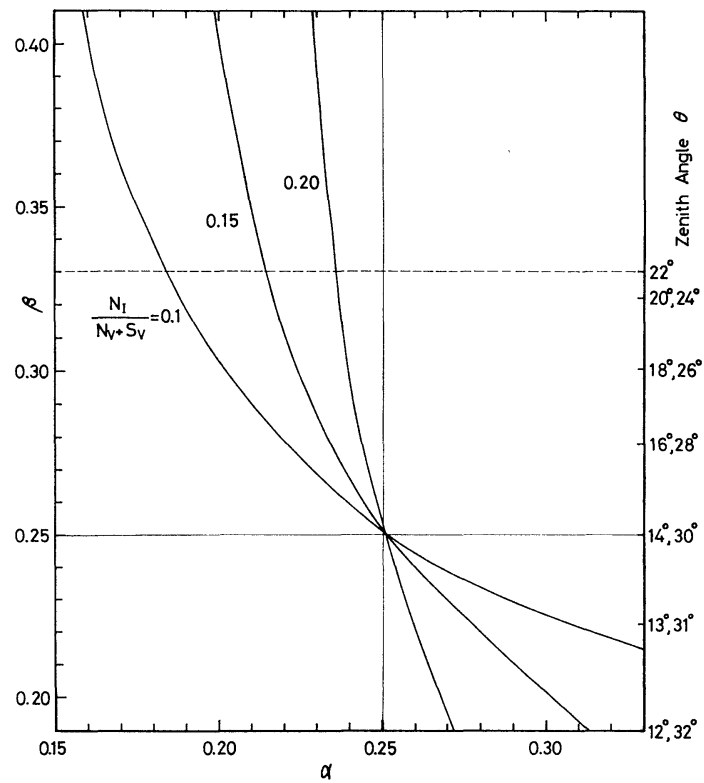

Fig. 16. Allowed regions of parameters $\alpha$ and $\beta$ for three different values of $N_{\mathrm{I}} /\left(N_{\mathrm{V}}+S_{\mathrm{V}}\right)$, and its corresponding zenith angle. 
The upper limit of $\alpha$ is the maximum value of $\bar{\alpha}(\theta)$ divided by $360^{\circ}$ and is 0.33 , while its lower limit corresponds to the extreme case in which the X-ray source is just out of the viewfield of the inclined counter. A similar situation applies to $\beta$.

From Eqs. (1) and (2), we obtain a relation between $\alpha$ and $\beta$. It is shown for three different values of $N_{\mathrm{I}} /\left(N_{\mathrm{V}}+S_{\mathrm{V}}\right)$ in Fig. 16. From Eq. (1) we find $\left(N_{\mathrm{I}}+S_{\mathrm{I}}\right) /\left(N_{\mathrm{V}}+S_{\mathrm{V}}\right)=0.25$, so that $\alpha$ equals to $\beta$ at 0.25 . It is found from Fig. 16 that the region allowable to $\alpha$ and $\beta$ is from zero to 0.33 . Since $\alpha$ can be deduced from $\bar{\alpha}(\theta)$ at a zenith angle $\theta$ as mentioned above, the value of $\alpha$ and hence $\beta$ are determined when $N_{\mathrm{I}} /\left(N_{\mathrm{v}}+S_{\mathrm{v}}\right)$ is given. The corresponding zenith angle $\theta$ is also given on the right-hand ordinate in Fig. 16.

\section{REFERENCES}

Barcus, J.R., R.R. Brown, R.H. Karas, K. Brønstad, H. Trefall, M. Kodama, and T.J. Rosenbers, Balloon observations of auroral-zone X-rays in conjugate regions, J. Atmos. Terr. Phys., 35, 497-511, 1973.

Beuerman, K.P., Secondary electrons and photons in the upper atmosphere, J. Geophys. Res., 76, 4291-4310, 1971.

Bradt, H., G. Garmire, M. Oda, G. Spada, B.V. Sreekantan, P. Gorenstein, and H. GURSKY, The modulation collimator in X-ray astronomy, Space Sci. Rev., 8, 471-506, 1968.

Brown, R.R., Electron precipitation in the auroral zone, Space Sci. Rev., 5, 311-387, 1966.

Gosling, J.T., Localization and motion of energetic electron precipitation during magnetic bays, J. Geophys. Res., 71, 835-848, 1966.

Horstman, H.M., G. Gavallo, and E. Moretti-Horstman, The $\mathrm{X}$ and $\gamma$ diffuse backgrounds, Rivista Nuovo Cimento, 5, 255-311, 1975.

IмноF, W.L., Review of rocket and satellite measurements of X-rays from electron precipitation at high latitudes, Proc. Int. Conf. ' $X$-Rays in Space,' Calgary, 2, 741-779, 1974.

KodAmA, M. and T. OGUTI, Spatial distributions of auroral zone X-rays as viewed from rocket altitudes, Mem. Natl. Inst. Polar Res., Ser. A, 14, 1-58, 1976.

OdA, M., Optical identification and interpretation of X-ray sources, Space Sci. Rev., 8, 507-533, 1968.

Ogawara, Y. and T. HAyAshi, Measurements of separation distances of rocket stages with r-rays, Proc. 9th Int. Symp. Space Tech. Sci., 659-665, 1971.

Okudaira, K., Apparent transformation of the X-ray source by Thomson scattering in the atmosphere, J. Geomag. Geoelectr., 1979 (to be published).

PARKs, G.K., Spatial characteristics of auroral-zone X-ray microbursts, J. Geophys. Res., 72, 215-226, 1967.

Peterson, L.E., Properties of individual X-ray sources, Non solar X-and gamma-ray astronomy, IAU Symp., No. 37, 59-80, 1969.

Rossi, B., High Energy Particles, p. 48, Prentice-Hall, 1952.

Trefall, H. and R.R. Brown, Report on balloon flights from northern Norway and Iceland 1971, SPARMO Bull., 5, 17-33, 1972.

Treilhou, J.P., Preliminary results of multi-balloon observations of X-rays in auroral conditions, Proc. Int. Conf. 'X-Rays in Space,' Calgary, 2, 1053-1068, 1974.

Venkatesan, K.K.V.D. and C.D. Anger, Investigation of electron precipitation during an auroral substorm by rocket-borne detectors, J. Geophys. Res., 80, 3205-3210, 1975. 